\title{
Dielectric Anomalies in Crystalline Ice: Indirect evidence of the Existence of a Liquid-liquid Critical Point in $\mathrm{H}_{2} \mathrm{O}$
}

Fei Yen ${ }^{1,2^{*}}$, Zhenhua $\mathrm{Chi}^{1,2}$, Adam Berlie ${ }^{1 \dagger}$, Xiaodi Liu ${ }^{1}$, Alexander F. Goncharov ${ }^{1,3}$

${ }^{1}$ Key Laboratory for Materials Physics, Institute of Solid State Physics, Hefei Institutes of Physical Science, Chinese Academy of Sciences, Hefei 230031, P. R.

China

${ }^{2}$ High Magnetic Field Laboratory, Hefei Institutes of Physical Science, Chinese Academy of Sciences, Hefei 230031, P. R. China

${ }^{3}$ Geophysical Laboratory, Carnegie Institution of Washington, 5251 Broad Branch Road, NW, Washington D.C., 20015, USA

*Correspondence: fyen18@hotmail.com

†'Present address: ISIS Neutron and Muon Source, STFC Rutherford Appleton Laboratory, Didcot, Oxfordshire OX11 0QX United Kingdom

"This document is the unedited Author's version of a Submitted Work that was subsequently accepted for publication in [The Journal of Physical Chemistry C], copyright (C) American Chemical Society after peer review. To access the final edited and published work see http://pubs.acs.org/doi/abs/10.1021/acs.jpcc.5b07635 " 


\begin{abstract}
The phase diagram of $\mathrm{H}_{2} \mathrm{O}$ is extremely complex; in particular, it is believed that a second critical point exists deep below the supercooled water (SCW) region where two liquids of different densities coexist. The problem however, is that SCW freezes at temperatures just above this hypothesized liquid-liquid critical point (LLCP) so direct experimental verification of its existence has yet to be realized. Here, we report two anomalies in the complex dielectric constant during warming in the form of a peak anomaly near $T_{\mathrm{p}}=203 \mathrm{~K}$ and a sharp minimum near $T_{\mathrm{m}}=212 \mathrm{~K}$ from ice samples prepared from SCW under hydrostatic pressures up to $760 \mathrm{MPa}$. The same features were observed about $4 \mathrm{~K}$ higher in heavy ice. $T_{\mathrm{p}}$ is believed to be associated to the nucleation process of metastable cubic ice $\mathrm{I} c$ and $T_{\mathrm{m}}$ the transitioning of ice $\mathrm{I} c$ to either ices $\mathrm{I} h$ or II depending on pressure. Given that $T_{\mathrm{p}}$ and $T_{\mathrm{m}}$ are nearly isothermal and present up to at least $620 \mathrm{MPa}$ and ending as a critical point near 33-50 $\mathrm{MPa}$, it is deduced that two types of SCW with different density concentrations exists which affects the surface energy of ice Ic nuclei in the "no man's land" region of the phase diagram. Our results are consistent with the LLCP theory and suggest that a metastable critical point exists in the region of $33-50 \mathrm{MPa}$ and $T_{\mathrm{c}} \geq 212 \mathrm{~K}$.
\end{abstract}

\title{
TOC Graphic:
}

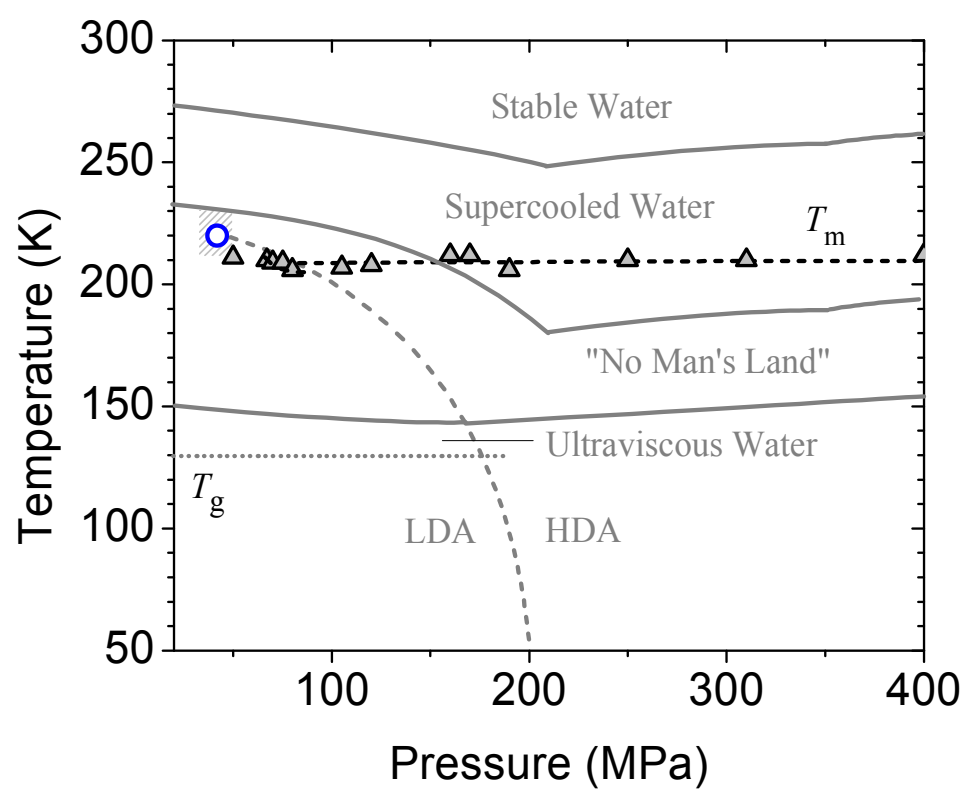

Keywords: liquid-liquid critical point, supercooled water, ice I $h$, ice Ic, ice II, dielectric constant, proton ordering. 
$\mathrm{H}_{2} \mathrm{O}$ ice is one of the most abundant solids in our universe with vast quantities existing on Earth and within our solar system. As such, many researchers are interested in knowing the structures, processes and patterns that these different ice phases exhibit. ${ }^{1}$ Depending on the temperature and pressure, the oxygen atoms in ice form different types of crystallographic configurations (Fig. 1) such as hexagonal (ice I), ${ }^{2}$ rhombohedral (ice II), ${ }^{3}$ tetragonal (ices III, IX and VI), ${ }^{4-6}$ monoclinic (ices V and XIII), ${ }^{7,8}$ other interpenetrating frameworks (ices VII and VIII), ${ }^{9,10}$ and even cubic (ice $\mathrm{X})$ at ultrahigh pressures. ${ }^{11,12}$ The hydrogen atoms (designated as the protons) on the other hand, are more flimsy and are only restricted by the ice rules. ${ }^{13}$ Namely, a) each proton is linked to two oxygen atoms, one via an intramolecular covalent bond and another via an intermolecular van der Waals hydrogen bond, and b) each oxygen atom is linked to four protons, two via covalent bonding and two via hydrogen bonding. At low temperatures the hydrogen atoms also form a crystallographic pattern like the oxygen atoms and the respective phases are considered to be "proton ordered". Much of the complexities in ice arise from the intra- and intermolecular bonding of the hydrogen atoms. ${ }^{14}$

Despite the simplicity of the $\mathrm{H}_{2} \mathrm{O}$ molecule, over a dozen different crystallographic phases (ices I $h$, Ic,${ }^{15}$ II-XV), at least three amorphous phases, ${ }^{16,17}$ a supercooled region, ${ }^{18}$ and a 'no man's land' occupy the phase diagram. ${ }^{19}$ Apart from the well established critical point where water and ice coexist, a second critical point is believed to reside near $210 \mathrm{~K}$ and $100 \mathrm{MPa}$ (Ref. ${ }^{20}$ ) separating two types of liquid structures composed of different concentrations of a low density liquid and a high 
density liquid. $^{21,22}$ The problem however, is that supercooled water (SCW) freezes at a temperature just above this hypothesized liquid-liquid critical point (LLCP) which makes it nearly impossible to access experimentally. As such, much work has been devoted on studying the metastable extensions of several phase boundary lines that extend toward the expected location of the LLCP, ${ }^{23,24,25}$ as well as nano-, ${ }^{26}$ and micro-sized ${ }^{27}$ samples to confirm the existence (or nonexistence) of the LLCP. As Stanley et al. propose, ${ }^{28}$ other indirect approaches are needed to confirm its existence which we attempt in this work. Verification of the existence of the LLCP will bring consistency to the phase behavior of metastable water and ice ${ }^{29}$ and further understand other systems that also possess a liquid-liquid phase boundary such as the cases in silicon, ${ }^{30,31}$ phosphorous ${ }^{32}$ and cerium. ${ }^{33}$

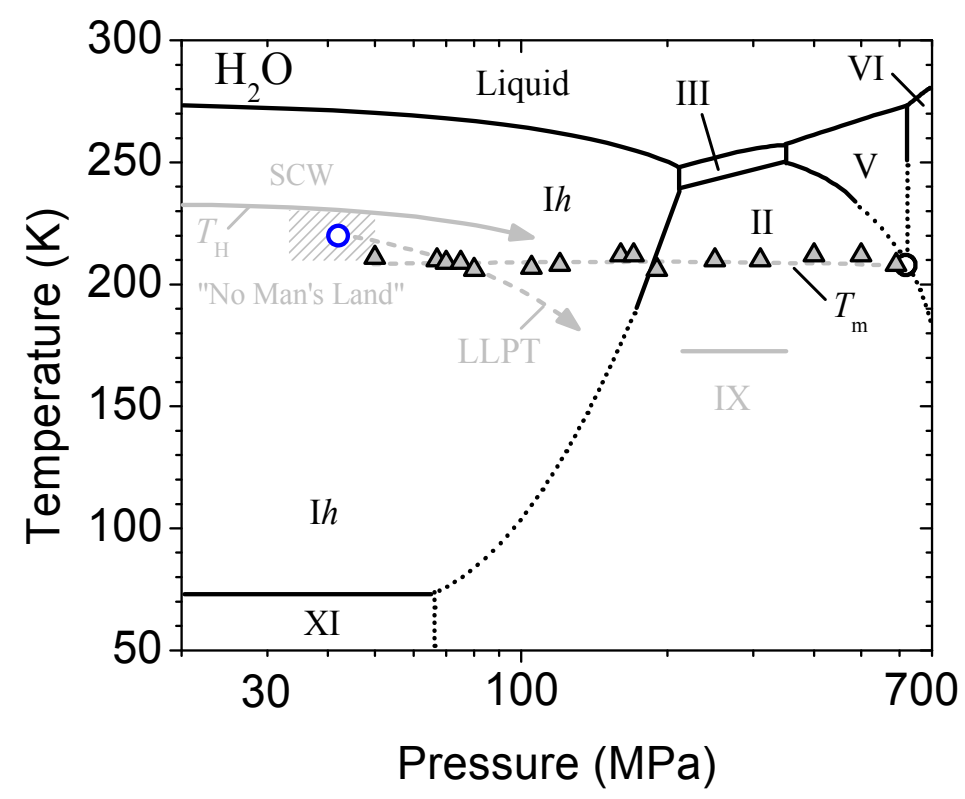

Fig. 1: Low pressure low temperature phase diagram of $\mathrm{H}_{2} \mathrm{O}$. All dotted lines and the triple point (black circle) near $620 \mathrm{MPa}$ and $208 \mathrm{~K}$ are based on guesswork. All phases and phase lines in grey are metastable. $T_{\mathrm{m}}$ (this work) represents the transition of metastable ice Ic into ices $\mathrm{I} h$ or II depending on pressure exhibited by a sharp minimum anomaly in the real and imaginary parts of the dielectric constant. $T_{\mathrm{m}}$ ends as a critical point somewhere in the region of $33<P_{\mathrm{c}}<50 \mathrm{MPa}$ and $210 \mathrm{~K}$ where we conjecture that this is the same critical pressure where a liquid-liquid phase transition (LLPT) line separating two types of liquid structures also end as suggested in Ref. ${ }^{20} \cdot T_{\mathrm{H}}$ 
is the homogeneous nucleation curve separating supercooled water (SCW) and "No Man's Land". Blue circle is plotted at $42 \mathrm{MPa}$ and $220 \mathrm{~K}$ (average of $210 \mathrm{~K}$ and the value of $T_{\mathrm{H}}$ at $42 \mathrm{MPa}$ ).

The dielectric constant is a macroscopic quantity that is proportional to the dipole moment distribution at the atomic level. Hence, any structural transition is readily reflected in the dielectric constant because the bond angles and lattice parameters which constitute the charge distributions undergo changes. During the proton (dis)ordering process, the imaginary part of the dielectric constant is particular sensitive since there are movement of charges involved. For this reason, we carried out high precision measurements of the complex dielectric constant on samples of $\mathrm{H}_{2} \mathrm{O}$ prepared from SCW as a function of temperature up to $760 \mathrm{MPa}$. We first present a detailed analysis of an isobar at $310 \mathrm{MPa}$ as an example to understand the different transformations that ice undergoes under pressure when subjected to cooling followed by warming. This will help appreciate the two new anomalies we identify in the form of a broadened peak near $T_{\mathrm{p}}=203 \mathrm{~K}$ followed by a sharp minimum near $T_{\mathrm{m}}=212 \mathrm{~K}$ during warming in the pressure range of $50-620 \mathrm{MPa}$. We attribute $T_{\mathrm{p}}$ to be associated to the nucleation process of small amounts of cubic ice (ice Ic) and $T_{\mathrm{m}}$ its subsequent transition to either ice I $h$ or II in the "no man's land" region of the metastable phase diagram of water. $T_{\mathrm{m}}$ is also intrinsic in $\mathrm{D}_{2} \mathrm{O}(\mathrm{D}=$ deuterium) and found to completely vanish at pressures below $33 \mathrm{MPa}$ suggesting that an LLCP indeed exists in 'no man's land'.

Figures $2 \mathrm{a}$ and $2 \mathrm{~b}$ show the real $\varepsilon^{\prime}(T)$ and imaginary parts $\varepsilon^{\prime \prime}(T)$ of the dielectric constant as a function of temperature at $310 \mathrm{MPa}$, respectively. At $T_{\mathrm{L}_{-} \mathrm{III}+\mathrm{SCW}}=252 \mathrm{~K}$ during cooling, part of the sample transformed into ice III represented by a shoulder 
type discontinuity in $\varepsilon^{\prime}(T)$ and $\varepsilon^{\prime \prime}(T)$ coinciding exactly with the $\mathrm{H}_{2} \mathrm{O}$ liquidus line. ${ }^{34}$ The residual liquid that did not transform remained as supercooled water (SCW) until $T_{\text {III }+ \text { SCW_III }}=232 \mathrm{~K}$ where heterogeneous nucleation of ice III occurred marked by a sharp drop in both $\varepsilon^{\prime}(T)$ and $\varepsilon^{\prime \prime}(T)$. This process is one method how crystalline ice III is formed. ${ }^{34}$ In the range of $211>T>164 \mathrm{~K}, \varepsilon^{\prime}(T)$ decreased by over half its value while $\varepsilon^{\prime \prime}(T)$ exhibited a minimum at $210 \mathrm{~K}$ and a maximum at $203 \mathrm{~K}$. The dielectric constant within an ice phase usually does not change by much so the origin of such a drastic change can be presumed to stem from proton ordering dynamics. Indeed, Whalley et al. concluded that continuous proton ordering occurs in ice III from $208 \mathrm{~K}$ to $163 \mathrm{~K}$ to end up forming ice IX. ${ }^{5}$

It was later confirmed by neutron diffraction on $\mathrm{D}_{2} \mathrm{O}$ (Refs. ${ }^{4,35}$ ) that only $96 \%$ of the deuterons ordered at $\sim 77 \mathrm{~K}$. According to DFT calculations from Ref. ${ }^{36}$, the protons should undergo a first-order phase transition to become a fully ordered antiferroelectric ground state at $T_{\mathrm{IX}}=126 \mathrm{~K}$. Unfortunately, Whalley et al., ${ }^{5}$ only presented data down to $158 \mathrm{~K}$ and the neutron diffraction studies were not carried out in situ nor under hydrostatic pressure conditions. In our case, a change of slope discontinuity was observed in our data in $\mathrm{d} \varepsilon^{\prime \prime}(T) / \mathrm{d} T$ (inset of Fig. 2b) which coincides exactly with $T_{\mathrm{IX}}$.

Upon warming, both $\varepsilon^{\prime}(T)$ and $\varepsilon^{\prime \prime}(T)$ took a different path from their cooling curves starting from $T>164 \mathrm{~K}$ which coincides with the fact that ice IX transforms into ice II upon warming. ${ }^{5}$ At $T_{\text {II_III }}=245 \mathrm{~K}$, a sharp step-up indicates the phase transition from ice II to ice III, also in good agreement with existing literature. ${ }^{34}$ The 
region below $T_{\text {III }+ \text { SCW_III }}=232 \mathrm{~K}$ of the cooling curve and above $T_{\text {II_III }}=245 \mathrm{~K}$ of the warming curve in both $\varepsilon^{\prime}(T)$ and $\varepsilon^{\prime \prime}(T)$ appear to be parts of the same function which is not surprising because in both of these two regions, the system was in the same ice III phase.

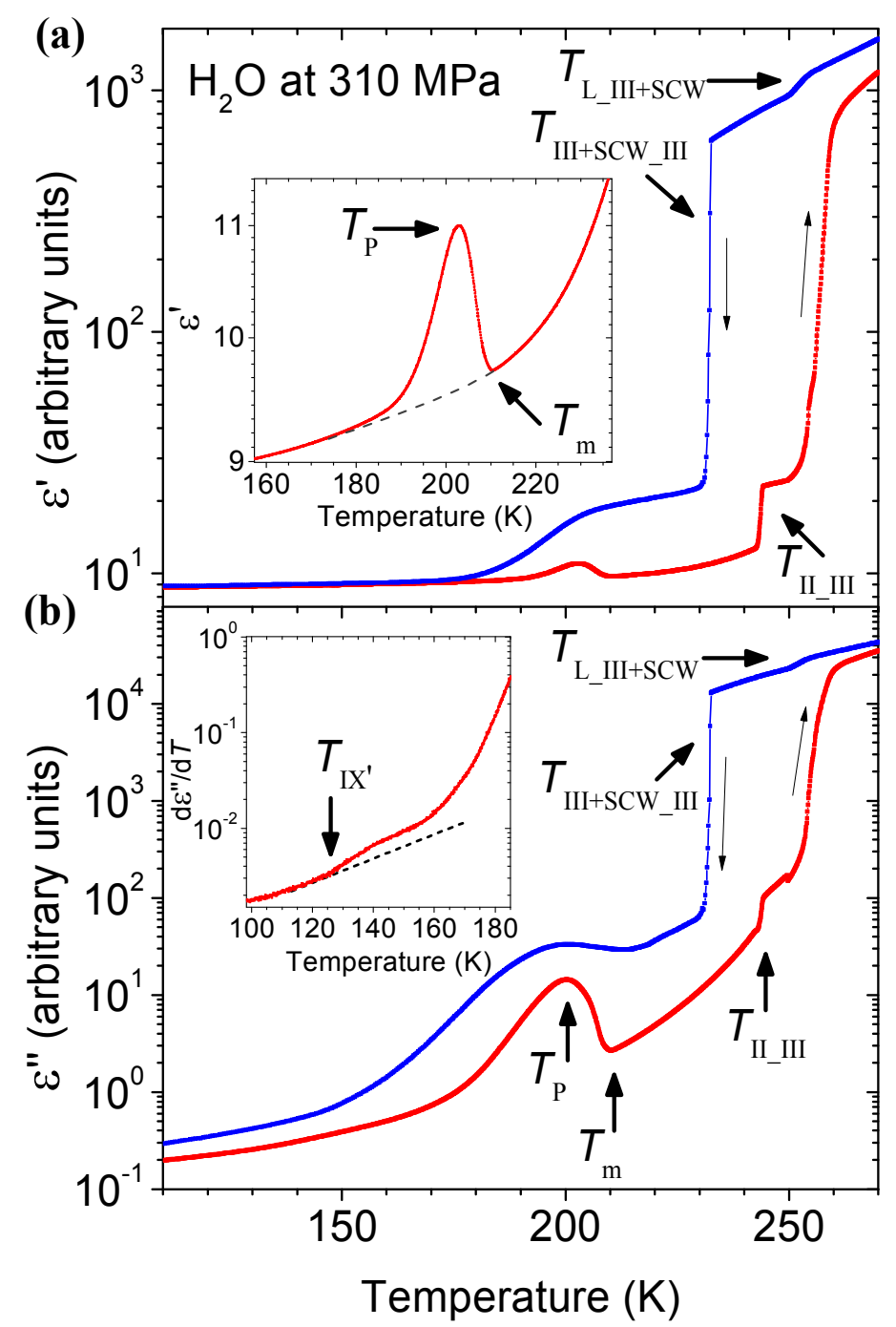

Fig. 2: Dielectric constant of $\mathrm{H}_{2} \mathrm{O}$ at $310 \mathrm{MPa}$. (a) Real $\varepsilon^{\prime}(T)$ and (b) imaginary $\varepsilon^{\prime \prime}(T)$ parts of the dielectric constant. At $T_{\mathrm{L}_{-} \mathrm{III}+\mathrm{SCW}}=252 \mathrm{~K}$, part of the sample transformed into ice III while the rest remained as supercooled water $(\mathrm{SCW})$. At $T_{\mathrm{III}+\mathrm{SCW} \_\mathrm{III}}=232 \mathrm{~K}$, the remaining $\mathrm{SCW}$ nucleated into ice III. At $T_{\text {II IIII }}=245 \mathrm{~K}$, the system transformed back to ice III. Two new anomalies are identified upon warming: a peak at $T_{\mathrm{p}}=203 \mathrm{~K}$ associated to the nucleation process of ice $\mathrm{I} c$; and a sharp minimum at $T_{\mathrm{m}}=210 \mathrm{~K}$ due to the transitioning of ice Ic into ice II. Inset of (b): An anomaly in the form of a linear to nonlinear change in $\mathrm{d} \varepsilon^{\prime \prime}(T) / \mathrm{d} T$ at $T_{\mathrm{IX}}=126 \mathrm{~K}$ suggests that full ordering of the protons occurred at $T<T_{\mathrm{IX}}$ according to Ref. ${ }^{36}$.

The new features we observe are a peak anomaly during warming in $\varepsilon^{\prime}(T)$ and 
$\varepsilon^{\prime \prime}(T)$ at $T_{\mathrm{p}}=203 \mathrm{~K}$ followed by a sharp minimum at $T_{\mathrm{m}}=210 \mathrm{~K}$ (inset of Fig. 2a and Fig. 2b). To understand more about the nature of $T_{\mathrm{m}}$, in one of the scans at $190 \mathrm{MPa}$, the temperature was lowered immediately after the sample passed through $T_{\mathrm{m}}$ (curve 3 in Fig. 3a). Interestingly, both $T_{\mathrm{p}}$ and $T_{\mathrm{m}}$ were not observed during cooling which classifies these two features as metastable. The temperature was lowered all the way down to $77 \mathrm{~K}$ followed by warming back to room temperature shown as curve 4 . Surprisingly, both $T_{\mathrm{p}}$ and $T_{\mathrm{m}}$ were also not observed during this second warming run hinting that these two anomalies may have been a consequence of the cooling process. It should be noted that a third cool down starting from room temperature and subsequently warmed up yielded the same results as curves 1 and 2.
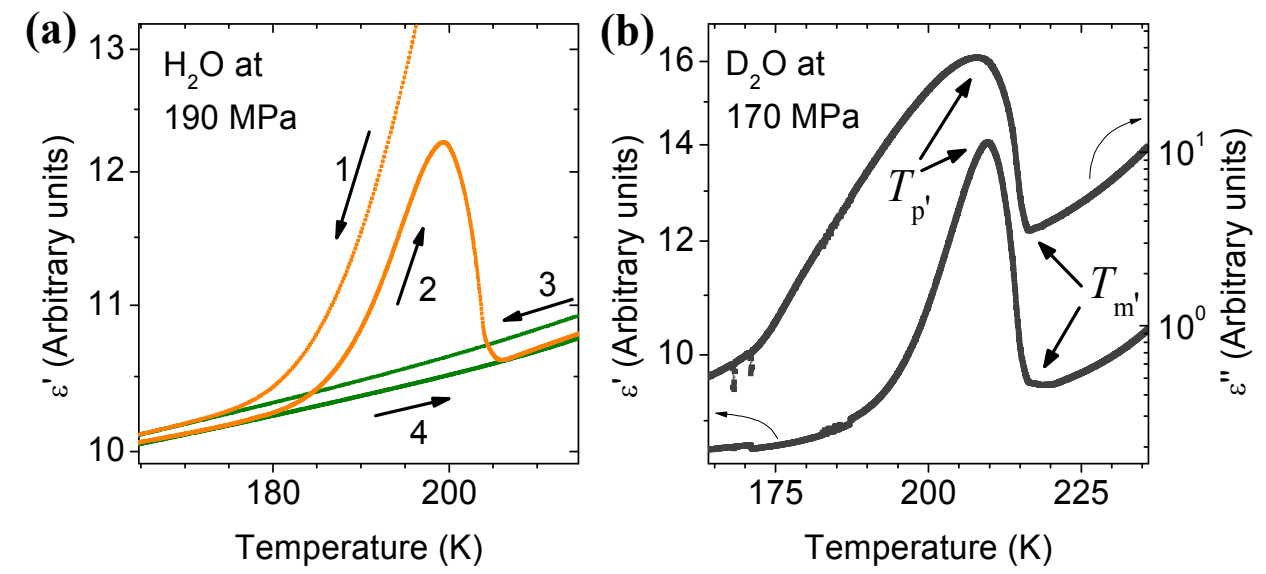

Fig. 3: (a) $\varepsilon^{\prime}(T)$ curves subjected to various initial conditions: curve 1 is the initial cool-down from $300 \mathrm{~K}$ to $77 \mathrm{~K}$, curve 2 is warming from 77 to $220 \mathrm{~K}$, curve 3 is cooling from $220 \mathrm{~K}$ back to $77 \mathrm{~K}$ and curve 4 is warming from 77 to $300 \mathrm{~K}$. (b) is $\varepsilon^{\prime}(T)$ and $\varepsilon^{\prime \prime}(T)$ in the case for $\mathrm{D}_{2} \mathrm{O}$ during warming at $170 \mathrm{MPa}$ where the two anomalies were also observed at $T_{\mathrm{p}}=208 \mathrm{~K}$ and $T_{\mathrm{m}}=216 \mathrm{~K}$.

Fig. $3 b$ shows $\varepsilon^{\prime}(T)$ and $\varepsilon^{\prime \prime}(T)$ for $\mathrm{D}_{2} \mathrm{O}$ at $170 \mathrm{MPa}$ where $T_{\mathrm{p}^{\prime}}=208 \mathrm{~K}$ and $T_{\mathrm{m}}=216$ K. The phase boundaries of $\mathrm{D}_{2} \mathrm{O}$ are usually about 4 degrees higher than $\mathrm{H}_{2} \mathrm{O}$ (Ref. ${ }^{37}$ ) so both sets of our results are in good agreement with each other.

Figures 4a-d show different warming curves of $\varepsilon^{\prime}(T)$ and $\varepsilon^{\prime \prime}(T)$ from 33 to 720 $\mathrm{MPa}$. In the low pressure region, the maximum in $T_{\mathrm{p}}$ and minimum in $T_{\mathrm{m}}$ were still 
clearly evident in the $70 \mathrm{MPa}$ curve. For the 67 and $50 \mathrm{MPa}$ curves, the maxima and minima features were diminished and evolved into a step anomaly. Finally at $33 \mathrm{MPa}$, $T_{\mathrm{p}}$ and $T_{\mathrm{m}}$ were undetected indicating the existence of a metastable critical point. At the high pressure end, $T_{\mathrm{m}}$ appears to intercept with the ice II/V/VI triple point estimated to reside near $620 \mathrm{MPa}$ and $208 \mathrm{~K} .^{38}$ It is not known if $T_{\mathrm{m}}$ and $T_{\mathrm{p}}$ completely vanish at $P>620 \mathrm{MPa}$ as they may have been masked by the dielectric constant of ice VI of which is the largest of all the ices.

During the freezing process multiple nucleation sites appear so grain boundaries will ultimately be present as the formation of one entire single crystalline piece is not probable. In between the crystalline regions pockets of topologically disordered material exist. We do not know the size of the pockets formed as the kinetics of ice nucleation in "no man's land" is still not well understood even at ambient pressure conditions. ${ }^{39,40}$ However, it is reasonable to presume that these pockets and interfaces are no more than a few nanometers in size. It is now well known that ice Ic is the most favorable type of ice to form in confined volumes and interfaces that are, respectively, 15 and $10 \mathrm{~nm}$ or smaller in the $160-220 \mathrm{~K}$ range. ${ }^{41,42}$ For instance, the type of ice that forms from supercooled water is a stacking type of ice Ic and ice $\mathrm{I} h$ with a ratio of about 2 to $1 .^{43}$

Interestingly, ice Ic has also been reported to form from amorphous ice ${ }^{44}$ and high pressure ices; ${ }^{45}$ its properties and degree of reconstruction of its stacking faults depend on the initial state of formation and surrounding temperature ${ }^{1}$ (meaning that different variants of ice Ic can exist). Specifically, ices II, III and V transform to ice Ic 
upon warming at ambient pressure in the ranges of $168-178 \mathrm{~K}, 148-158 \mathrm{~K}$ and 151-155 K, respectively. ${ }^{45}$ Upon further warming, ice Ic transforms into ice $\mathrm{I} h$ at $180-228 \mathrm{~K} \cdot{ }^{46,47}$ The reason why metastable ice Ic forms at low temperature is because the surface energy of its nuclei is lower than ice $\mathrm{I} h{ }^{48}$ However, at higher temperature, nucleation of ice $\mathrm{I} h$ is favored because the surface entropy of its nuclei is larger than that of ice Ic. ${ }^{49}$ Thus, we attribute $T_{\mathrm{p}}$ to be associated to the nucleation process of ice Ic of which its nuclei most likely formed during the cooling process which explains why a minimum in $\varepsilon^{\prime \prime}(T)$ exists near $203 \mathrm{~K}$ in the cooling curves (Fig. 2b). This is key toward understanding the kinetics of ice nucleation in "no man's land" as we are merely in the beginning stages of understanding its details in SCW. ${ }^{50}$ Hence, $T_{\mathrm{p}}$ marks the point where the sample comprised the highest amount of ice Ic; and $T_{\mathrm{m}}$ the abrupt transformation of ice Ic to either ice $\mathrm{I} h$ or ice II depending on the pressure environment. Note that ice Ic is metastable in the phase spaces of ices I $h$ and II so once ice Ic transitions into ices $\mathrm{I} h$ or II upon warming, it can no longer transition back; this is consistent with the metastable behavior of $T_{\mathrm{m}}$ and $T_{\mathrm{p}}$ (Fig. 3a). For instance, in Fig. 2a, ice Ic nucleated into ice II at $T_{\mathrm{m}}$ so in the region of $T_{\mathrm{m}}<T<245 \mathrm{~K}$ the entire sample was in the ice II phase.

It should also be noted that ice Ic has only been reported to form from quenching $^{42}$ with the slowest cooling rate being $10 \mathrm{~K}-\min ^{-1}$ (Ref. ${ }^{47}$ ) at ambient conditions. To our knowledge, no work on ice Ic under pressure has ever been reported. In our work, our cooling rates have been at most $3 \mathrm{~K}-\mathrm{min}^{-1}$ so formation of ice $\mathrm{I} c$ is not expected near ambient pressure conditions which is consistent with the 
fact that $T_{\mathrm{p}}$ and $T_{\mathrm{m}}$ are absent up to $33 \mathrm{MPa}$. Only starting from $50 \mathrm{MPa}$ do $T_{\mathrm{p}}$ and $T_{\mathrm{m}}$ appear. Pressure somehow favors the formation of ice Ic when cooled slowly either by lowering the surface energy of its nuclei or by further reducing the volumes of the pockets of trapped disordered material. Starting from $50 \mathrm{MPa}$ the $T_{\mathrm{p}}$ and $T_{\mathrm{m}}$ features grow with pressure; however, their critical temperatures remain nearly unchanged for a large range of pressure which suggests that there are two types of liquid structures that affect the surface energy of ice Ic nuclei differently. If we take into account that the LLCP is situated in the vicinity near $100 \mathrm{MPa}$ and $220 \mathrm{~K}$ as originally suggested in Ref. ${ }^{20}$, and that a liquid-liquid phase transition (LLPT) line extends out of the LLCP toward lower temperature and higher pressure separating a low pressure and a high pressure liquid structure (Fig. 1), an indirect correlation can be drawn between the LLCP and the critical point obtained in this work.

Below the homogenous nucleation curve $T_{\mathrm{H}}$, it is not energetically favorable for $\mathrm{H}_{2} \mathrm{O}$ to exist in liquid form. The properties of ice $\mathrm{I} c$ are unique in that it is more fluid-like than all of the other crystalline phases as its stacking faults can be reconfigured depending on the surrounding environment. ${ }^{1}$ Hence, out of all the possible ice phases, ice Ic appears to be the only phase that would be susceptible enough to changes affecting its nucleation process should there be two different types of liquid structures residing below $T_{\mathrm{H}}$. Based on the correlation drawn between the LLCP and the critical point identified in this work, we suggest that the critical pressure of the LLCP resides in the pressure range of $33<P_{\mathrm{c}}<50 \mathrm{MPa}$. As for the critical temperature, we suggest that $T_{\mathrm{H}}>T_{\mathrm{c}} \geq T_{\mathrm{m}}$ since $T_{\mathrm{m}}$ is only representative of the 
transition temperature of ice Ic to ices I $h$ or II. The deduced pressure and temperature ranges are drawn as a grey box in Fig. 1 along with its average values marked as a blue circle at $42 \mathrm{MPa}$ and $220 \mathrm{~K}$. The obtained values for $P_{\mathrm{c}}$ and $T_{\mathrm{c}}$ are in agreement with many of the values extracted from indirect experiments ${ }^{23-25,51}$ and predicted by recent simulations models. ${ }^{52-55}$ 

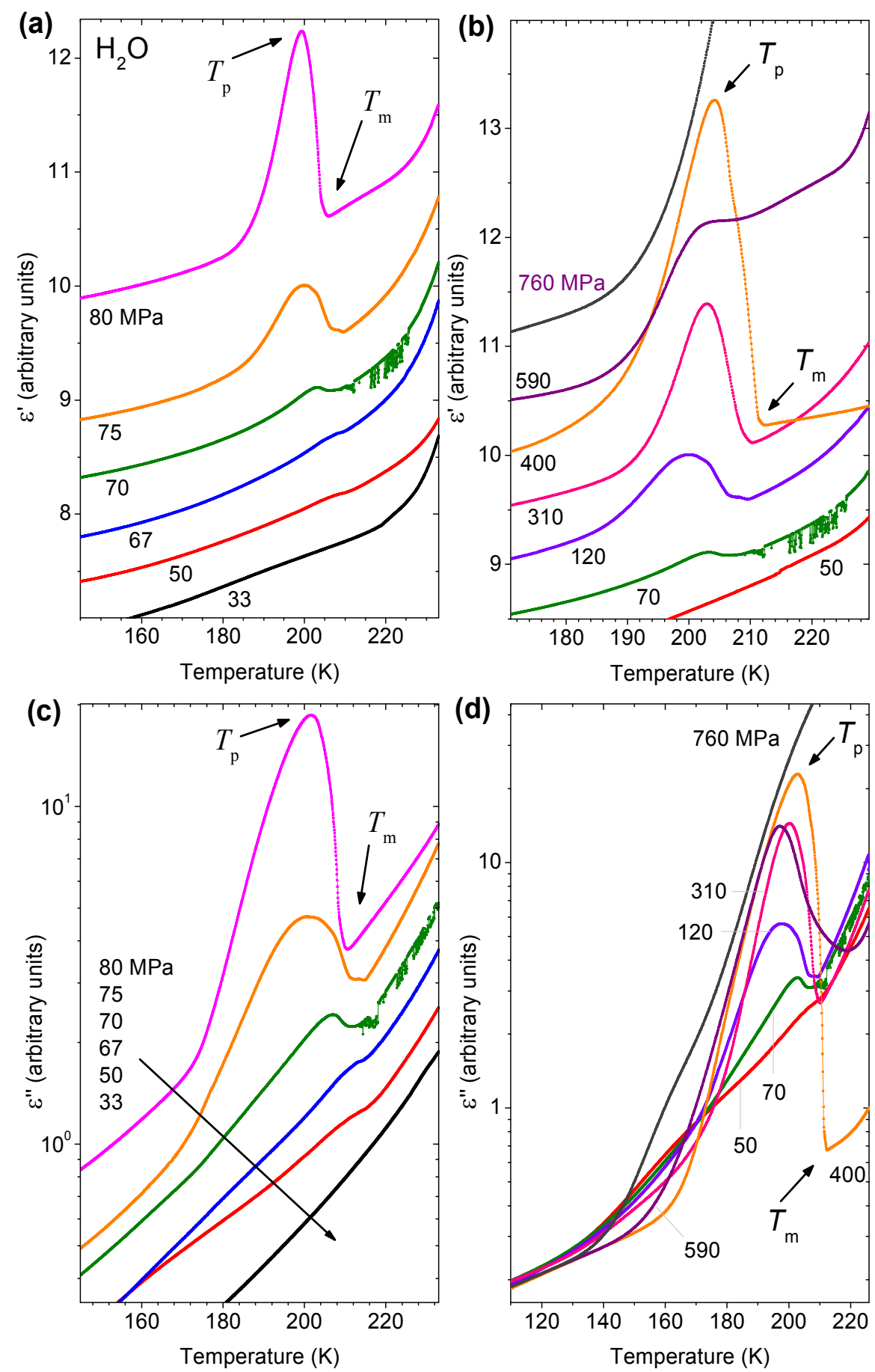

Fig. 4: Dependence of $T_{\mathrm{p}}$ and $T_{\mathrm{m}}$ in $\varepsilon^{\prime}(T)$ and $\varepsilon^{\prime \prime}(T)$ at different hydrostatic pressure. The 110 and $400 \mathrm{MPa}$ isobars were from a different sample. The $T_{\mathrm{m}}$ values at different pressure are plotted in Fig. 1.

This work was made possible in part via the support of the National Science Foundation of China grant numbers 11374307 and 51372249, and the Director's Grants of the Hefei Institutes of Physical Science, Chinese Academy of Sciences grant number YZJJ201313. 


\section{Methods:}

The samples were prepared by pressurizing deionized and degassed, liquid $\mathrm{H}_{2} \mathrm{O}$ (Milli-Q Direct 8) to the desired pressure at room temperature with a $\mathrm{BeCu}$ clamp cell then cooled to $77 \mathrm{~K}$ at $\sim 2-3 \mathrm{~K}-\mathrm{min}^{-1}$ via a customized gas exchange cryostat. For the $\mathrm{D}_{2} \mathrm{O}$ experiments, $99.9 \%$ isotopic purity from Sigma Aldrich) was used and the same procedure was followed. The real and imaginary parts of the dielectric constant were obtained by measuring the capacitance and loss tangent, respectively, of a pair of Pt electrodes in the form of parallel plates at $1 \mathrm{kHz}$ with an Andeen Hagerleen (AH2500A) capacitance bridge. The electrodes were dipped inside a Teflon capsule filled with liquid $\mathrm{H}_{2} \mathrm{O}$ so the sample itself was also the pressure medium. More details can be found elsewhere. ${ }^{56}$ Force was applied only when the sample was in liquid form so hydrostatic conditions were maintained upon ice nucleation.

\section{References:}

(1) Bartels-Rausch, T.; Bergeron, V.; Cartwright, J. H. E.; Escribano, R.; Finney, J. L.; Grothe, H.; Gutiérrez, P. J.; Haapala, J.; Kuhs, W. F.; Pettersson, J. B. C.; et al. Ice Structures, Patterns, and Processes: A View Across the Icefields. Rev. Mod. Phys. 2012, 84, 885-944.

(2) Bjerrum, N. Structure and Properties of Ice. Science 1952, 115, 385-390.

(3) Kamb, B. Ice II: A Proton-Ordered Form of Ice. Acta Crystallogr. 1964, 17, 1437-1449.

(4) Londono, J. D.; Kuhs, W. F.; Finney, J. L. Neutron Diffraction Studies of Ices III and IX on Under-Pressure and Recovered Samples. J. Chem. Phys. 1993, 98, 4878-4888.

(5) Whalley, E.; Heath, J. B. R.; Davidson, D. W. Ice IX: An Antiferroelectric Phase Related to Ice III. J. Chem. Phys. 1968, 48, 5, 2362-2370.

(6) Kuhs, W. F.; Finney, J. L.; Vettier, C.; Bliss, D. V. Structure and Hydrogen Ordering in Ices VI, VII and VIII by Neutron Powder Diffraction. J. Chem. Phys. 1984, 81, 3612-3623.

(7) Kamb, B.; Prakash, A.; Knobler, C. Structure of ice V. Acta Crystallogr. 1967, 22, 706-715.

(8) Salzmann, C. G.; Radaelli, P. G.; Hallbrucker, A.; Mayer, E.; Finney, J. L. The Preparation and Structures of Hydrogen Ordered Phases of Ice. Science 2006, 311, 1758-1761. 
(9) Jorgensen, J. D.; Worlton, T. G. Disordered Structure of $\mathrm{D}_{2} \mathrm{O}$ Ice VII from in situ Neutron Powder Diffraction. J. Chem. Phys. 1985, 83, 329-333.

(10) Besson, J. M.; Pruzan, P.; Klotz, S.; Hamel, G.; Silvi, B.; Nelmes, R. J.; Loveday, J. S.; Wilson, R. M.; Hull, S. Variation of Interatomic Distances in Ice-VIII to 10 GPa. Phys. Rev. B 1994, 49, 12540-12550.

(11) Hemley, R. J.; Jephcoat, A. P.; Mao, H. K.; Zha, C. S.; Finger, L. W.; Cox, D. E. Static Compression of $\mathrm{H}_{2} \mathrm{O}$-Ice to $128 \mathrm{GPa}$ (1.28 Mbar). Nature 1987, 330, 737-740.

(12) Goncharov, A. F.; Goldman, N.; Fried, L. E.; Crowhurst, J. C.; Kuo, I-F. W.; Mundy, C. J.; Zaug, J. M. Dynamic Ionization of Water under Extreme Conditions. Phys. Rev. Lett. 2005, 94, 125508-4.

(13) Bernal, J. D.; Fowler, R. H. A Theory of Water and Ionic Solution with Particular Reference to Hydrogen and Hydroxyl Ions. J. Phys. Chem. 1933, 1, 515-548.

(14) Stokely K.; Mazzaa, M. G.; Stanley, H. E.; Franzese, G. Effect of Hydrogen Bond Cooperativity on the Behavior of Water. Proc. Nat. Acad. Sci. 2010, 107, 1301-1306.

(15) Dowell, L. G.; Rinfret, A. P. Low-Temperature Forms of Ice as Studied by X-Ray Diffraction. Nature 1960, 188, 1144-1148.

(16) Mishima, O. Reversible First-Order Transition between Two $\mathrm{H}_{2} \mathrm{O}$ Amorphs at $\sim 0.2 \mathrm{GPa}$ and $\sim 135$ K. J. Chem. Phys. 1994, 100, 5910-5912.

(17) Loerting, T.; Salzmann, C.; Kohl, I.; Meyer, E.; Hallbrucker, A. A Second Distinct Structural "State" of High-Density Amorphous Ice at $77 \mathrm{~K}$ and 1 bar. Phys. Chem. Chem. Phys. 2001, 3, 5355-5357.

(18) Angell, C. A. Supercooled Water. Ann. Rev. Phys. Chem. 1983, 34, 593-630.

(19) M. Chaplin.www1.lsbu.ac.uk/water/amorphous_ice.html

(20) Poole, P. H.; Sciortino, F.; Essmann, U.; Stanley, H. E. Phase Behavior of Metastable Water. Nature 1992, 360, 324-328.

(21) Bertrand, C. E.; Anisimov, M. A. Peculiar Thermodynamics of the Second Critical Point in Supercooled Water. J. Phys. Chem. B 2011, 115, 14099-14111.

(22) Holten, V.; Anisimov, M. A. Entropy-Driven Liquid-Liquid separation in Supercooled Water. Sci. Rep. 2012, 2, 713, 1-7.

(23) Mishima, O.; Stanley, H. E. The Relationship between Liquid, Supercooled, and Glassy Water. Nature 1998, 396, 329-335.

(24) Mishima, O.; Stanley, H. E. Decompression-Induced Melting of Ice IV and the Liquid-Liquid Transition in Water. Nature 1998, 392, 164-168.

(25) Mishima, O. Liquid-Liquid Critical Point in Heavy Water, Phys. Rev. Lett. 2000, 85, 334-336.

(26) Mallamace, G.; Corsaro, C.; Baglioni, P.; Fratini, E.; Chen, S.-H. The Dynamical Crossover Phenomenon in Bulk Water, Confined Water and Protein Hydration Water. J. Phys. Condens. Matt. 2012, 24, 064103.

(27) Sellberg, J. A.; Huang, C.; McQueen, T. A.; Loh, N. D.; Laksmono, H.; Schlesinger, D.; Sierra, R.G.; Nordlund, D.; Hampton, C. Y.; Starodub, D.; et al. Ultrafast X-Ray Probing of Water Structure below the Homogeneous Ice Nucleation Temperature. Nature 2014, 510, 381-384.

(28) Stanley, H. E.; Buldyrev, S. V.; Franzese, G.; Giovambattista, N.; Starr, F. W. Static and Dynamic Heterogeneities in Water. Phil. Trans. R. Soc. A 2005, 363, 509-523.

(29) Debenedetti, P. G. Supercooled and Glassy Water. J. Phys.: Condens. Mat. 2003, 15, R1669-R1726. 
(30) Sastry, S.; Angell, C. A. Liquid-Liquid Phase Transition in Supercooled Silicon. Nat. Mater. 2003, 2, 739-743.

(31) Vasisht, V. V.; Saw, S.; Sastry, S. Liquid-Liquid Critical Point in Supercooled Silicon. Nat. Phys. 2011, 7, 549-553.

(32) Katayama, Y.; Mizutani, T.; Utsumi, W.; Shimomura, O.; Yamakata, M.; Funakoshi, K. A First-Order Liquid-Liquid Phase Transition in Phosphorus. Nature 2000, 403, 170-173.

(33) Cadient, A.; Hu, Q. Y.; Meng, Y.; Cheng, Y. Q.; Chen, M. W.; Shu, J. F.; Mao, H. K.; Sheng, H. W. First-Order Liquid-Liquid Phase Transition in Cerium. Phys .Rev. Lett. 2013, 110, 125503.

(34) Bridgman, P. W. Water, in the Liquid and Five Solid Forms, under Pressure. Proc. Am. Acad. Arts Sci. 1912, 47, 441-558.

(35) La-Placa, S. J.; Hamilton, W. C.; Kamb, B.; Prakash, A. On a Nearly Proton Ordered Structure for Ice IX. J. Chem. Phys. 1973, 58, 567-580.

(36) Knight, C.; Singer, S. J. A Reexamination of the Ice III/IX Hydrogen Bond Ordering Phase Transition. J. Chem. Phys. 2006, 125, 064506-10.

(37) Bridgman, P. W. The Pressure-Volume-Temperature Relations of the Liquid, and the Phase Diagram of Heavy Water. J. Chem. Phys. 1935, 3, 597-605.

(38) Durham, W. B.; Heard, H. C.; Kirby, S. H. Experimental Deformation of Polycrystalline $\mathrm{H}_{2} \mathrm{O}$ Ice at High Pressure and Low Temperature: Preliminary Results. J. Geophys. Res. 1983, 88, B377-B392.

(39) Yan, J. Y.; Patey, G. N. Heterogeneous Ice Nucleation Induced by Electric Fields. J. Phys. Chem. Lett. 2011, 2, 2555-2559.

(40) Laksmono, H.; McQueen, T. A.; Sellberg, J. A.; Loh, N. D.; Huang, C.; Schlesinger, D.; Sierra, R. G.; Hampton, C. Y.; Nordlund, D.; Beye, M.; et al. Anomalous Behavior of the Homogeneous Ice Nucleation Rate in "No-Man's Land". J. Phys. Chem. Lett. 2015, 6, 2826-2832.

(41) Johari, G. P. Water's Size-Dependent Freezing to Cubic Ice. J. Chem. Phys. 2005, 122, 194504-5.

(42) Mayer, E.; Hallbrucker, A. Cubic Ice from Liquid Water. Nature 1987, 325, 601-602.

(43) Moore, E. B.; Moliner, V. Is it Cubic? Ice Crystallization from Deeply Supercooled Water. Phys. Chem. Chem. Phys. 2011, 13, 20008-20016.

(44) Dowell, L. G.; Rinfret, A. P. Low-Temperature Forms of Ice as Studied by X-Ray Diffraction. Nature 1960, 188, 1144-1148.

(45) Bertie, J. E.; Calver, L. D.; Whalley, E. Transformations of Ice II, Ice III and Ice V at Atmospheric Pressure. J. Chem. Phys. 1963, 38, 840-846.

(46) Raza, Z,; Alfe, D.; Salzmann, C. G.; Klimes, J.; Michaelides, A.; Slater, B. Proton Ordering in Cubic Ice and Hexagonal Ice; a Potential New Ice Phase--XIc. Phys. Chem. Chem. Phys. 2011, 13, 19788-19795.

(47) Murray, B. J.; Bertram, A. K. Formation and Stability of Cubic Ice in Water Droplets. Phys. Chem. Chem. Phys. 2006, 8, 186-192.

(48) Takahashi, T. On the Role of Cubic Structure in Ice Nucleation. J. Cryst. Growth 1982, 59, 441-449.

(49) Kiefte, H; Clouter, M. J.; Whalley, E. Cubic Ice, Snowflakes, and Rare-Gas Solids: Surface Energy, Entropy and the Stability of Small Crystals. J. Chem. Phys. 1984, 81, 1419-1420. 
(50) Sanz, E.; Vega, C.; Espinosa, J. R.; Caballero-Bernal, R.; Abascal, J. L. F.; Valeriani, C. Homogeneous Ice Nucleation at Moderate Supercooling from Molecular Simulation. J. Am. Chem. Soc. 2013, 135, 15008-15017.

(51) Mishima, O. Volume of Supercooled Water under Pressure and the Liquid-Liquid Critical Point. J. Chem. Phys. 2010, 133, 144503-6.

(52) Fuentevilla, D. A.; Anisimov, M. A. Scaled Equation of State for Supercooled Water near the Liquid-Liquid Critical Point. Phys. Rev. Lett. 2006, 97, 195702-4.

(53) Holten, V.; Palmer, J. C.; Poole, P. H.; Debenedetti, P. G.; Anisimov, M. A. Two-State Thermodynamics of the ST2 Model for Supercooled Water. J. Chem. Phys. 2014, 140, 104502-12. (54) Holten, V.; Bertrand, C. E.; Anisimov, M. A.; J. V. Sengers. Thermodynamics of Supercooled Water. J. Chem. Phys. 2012, 136, 094507.

(55) Chiu, J.; Starr, F. W.; Giovambattista, N. Heating-Induced Glass-Glass and Glass-Liquid Transformations in Computer Simulations of Water. J. Chem. Phys. 2014, 140, 114504-13.

(56) Yen, F.; Chi, Z. H. Proton Ordering Dynamics of $\mathrm{H}_{2} \mathrm{O}$ Ice. Phys. Chem. Chem. Phys., 2015, $17,12458-12461$. 